\title{
Perspectives historiques et contemporaines sur l'occulte dans la correspondance Freud-Ferenczi
}

Thomas Rabeyron, Docteur en Psychologie Clinique, Psychologue Clinicien, Attaché Temporaire d'Enseignement et de Recherche, Université Lumière Lyon II

Renaud Evrard, Psychologue Clinicien, Doctorant en Psychologie Clinique, Université de Rouen

\section{Résumé}

La correspondance Freud-Ferenczi met en lumière leurs intérêts réciproques pour la transmission de pensée et la façon dont ils ont partagé, dans l'intimité de ces lettres, leurs expériences et leurs réflexions respectives à ce sujet. Si Ferenczi fut l'instigateur principal des échanges sur la transmission de pensée, Freud proposa également dans cette correspondance ses premières réflexions concernant ses deux cas majeurs sur la télépathie: l'empoisonnement par les huitres et la prophétie des jumeaux. Nous proposons dans cet article une lecture historique détaillée de ces échanges de façon à montrer leur actualité et leur pertinence, en particulier dans la compréhension de certains processus psychiques en jeu dans la clinique contemporaine des expériences exceptionnelles.

Mots clés : transfert de pensée, télépathie, co-pensée, transfert, contre-transfert.

\section{Historical and contemporary perspectives about occultism in Freud- Ferenczi correspondence}

\section{Abstract}

Correspondence between Freud and Ferenczi sheds light on their common interest about thought transference and the way they have shared in the intimacy of these letters, their experiences and their personal thinking about this topic. If Ferenczi has been the main investigator of these writings about though transference, Freud has also proposed in this correspondence his first reflections concerning his two major cases about telepathy: the oysters poisoning and the twins prophecy. We propose in this paper an historical and detailed analysis of this correspondence in order to show his current relevance, especially in the understanding of some psychological processes concerning contemporary clinical aspects of exceptional experiences.

Keywords : thought transference, telepathy, co-thinking, transference, countertransference 


\section{L'occulte et les origines de la psychanalyse}

L'atmosphère intellectuelle dans laquelle la psychanalyse fut inventée était riche de potentialités dont certaines se retrouvent aujourd'hui abandonnées ou marginalisées. C'est ainsi, comme le remarque Régine Plas, que les premiers psychologues ont

«presque tous été, de près ou de loin, impliqués dans des recherches qui seraient, de nos jours, exclues du champ de la psychologie et renvoyées à la parapsychologie comme, par exemple, l'étude de ces phénomènes que l'on appelle actuellement " perception extra-sensorielle " ou " télépathie ". » (Plas, 2000, p. 16)

Freud s'y intéressa également, si bien que lorsqu'il s'occupa des théories sur le rêve, il proposa notamment une interprétation de leur « signification occulte » (dans un chapitre qui aurait dû être publié dans l'édition définitive de la Traumdeutung, Freud, 1925), à propos d'un rêve apparemment "prémonitoire » d'une patiente (Freud, 1899). Cet intérêt de Freud lui a cependant été reproché par certains qui le considèrent comme la conséquence d'un mélange de superstition et de naïveté (Onfray, 2010). Des historiens ont pourtant mis en évidence la rigueur avec laquelle Freud avait abordé le champ de l'occultisme (Moreau, 1976 ; Méheust, 1999) et il nous semble également qu'une lecture attentive des écrits de Freud sur ce sujet montre la pertinence de ses réflexions sur ce thème. Il a ainsi évolué d'un scepticisme ferme vers une ouverture limitée et stratégique, concernant le transfert de pensée. Nous souhaiterions dans ce travail restituer précisément ce que fut l'attitude du fondateur de la psychanalyse, et l'explorer davantage à partir de l'analyse de la correspondance complète entre Freud et Ferenczi.

Cette imprégnation par les questions liées à l'occulte fut encore plus marquant pour Ferenczi et Jung, puisqu'il les mit indirectement sur la voie d'une science de l'activité inconsciente. Ainsi, Ferenczi s'était imprégné très jeune des ouvrages sur les «sciences psychiques ${ }^{i}$. Sa première publication (Ferenczi, 1899), dans une revue médicale hongroise, fut consacrée au spiritisme. Le choix de la revue lui fut même dicté lors d'une crise d'écriture automatique (Fodor, 1979)! Quant à Jung, sa thèse de 1902 portait sur la psychologie et la psychopathologie des phénomènes dits occultes, thème qu'il explorera durant toute sa carrière.

Or, les historiens ont montré comment, loin de répudier leurs intérêts antérieurs, ceux qui devinrent les plus proches disciples de Freud le pressèrent de s'occuper de ce sujet. Gori (1996) a analysé cette situation sous l'angle des relations transférentielles entre disciples et maître. Il analyse les intérêts de Ferenczi et Jung pour le transfert de pensée comme des acting out adressés à Freud, en l'absence d'un véritable cadre didactique. Si bien que la correspondance qui nous occupe joue comme révélateur de l'inachevé de la relation analytique, en particulier par l'expression des pensées de transfert sous couvert du transfert de pensée.

La correspondance Freud-Ferenczi, qui s'étend de 1908 à 1933, disponible en trois volumes (Freud \& Ferenczi, 1994, 1996a, 1996b), offre ainsi un éclairage intéressant sur les difficultés rencontrées par les deux psychanalystes face à l'occultisme et la transmission de pensée, difficultés qui sont toujours d'actualité aujourd'hui et dont les prémisses permettent notamment de mieux éclairer la complexité de certaines expériences spécifiques, reléguées habituellement dans le champ du "paranormal» et que nous avons proposé d'appeler « expériences exceptionnelles » (Rabeyron, 2009 ; Rabeyron, Chouvier \& Le Maléfan, 2010). Nous souhaiterions ainsi analyser de façon détaillée cette correspondance dans la continuité de plusieurs travaux déjà existants sur ce thème (Gori, 1996; Widlöcher, 2004 ; Turnheim, 2008) 


\title{
2. Analyse de la correspondance Freud-Ferenczi
}

\subsection{Premiers échanges : déjà-vu, métempsychose et Mme Seidler}

Nous trouvons une première allusion à l'occultisme sous la plume de Ferenczi décrivant, le 5 mai 1909, l'analyse d'un «jeune homme, très intelligent, homosexuel » chez qui le « déjà-vu » est « frappant » :

\begin{abstract}
« Il raconte ensuite les événements du jour et, entre autres, un "déjà-vu" ; puis, au cours de l'analyse du rêve qui suit, un fragment de rêve oublié apparaît, qui lui permet de comprendre cette sensation de familiarité de façon immédiate, ou seulement au cours de l'interprétation. Cette "autre vie d'il y a plusieurs milliers d'années", c'était la vie onirique de la nuit précédente, étroitement associée, il est vrai, à des impressions de l'enfance depuis longtemps oubliées. La croyance si répandue à la renaissance et à la métempsychose n'admettrait-elle pas la même explication? » (Freud \& Ferenczi, 1994, p. 69)
\end{abstract}

Ferenczi propose une brève analyse du phénomène de déjà-vu qu'il associe à la croyance à la réincarnation ou métempsychose, avec une hypothèse dynamique : le retour d'éléments oniriques et d'impressions de l'enfance. Il publiera ce cas (Ferenczi, 1912), amenant Freud à compléter son explication psychanalytique du déjà-vu ou déjà-vécu (Freud, 1914, 1936) qu'il réduisait auparavant au seul déjà-rêvé (Freud, 1901).

Cinq mois plus tard, de retour de leur voyage aux États-Unis, Freud et Ferenczi rendent visite à la voyante berlinoise Frau Seidler, qui parvint à deviner que le courrier qui lui était présenté provenait de Vienne, ce que Freud qualifiera de «don physiologique » ${ }^{\mathrm{ii}}$. Ferenczi va poursuivre les expériences et, le 5 octobre 1909, il écrit qu'il a demandé à la voyante de décrire les personnalités du Professeur Philipp et de Freud, à partir d'une carte adressée par ce dernier :

«Lorsque je cherche à m'expliquer ce que j'ai vu et entendu, je dois reconnaître que je n'en suis pas capable. Lire à travers un foulard, c'est-à-dire les yeux bandés, ce peut être de la prestidigitation. Mais deviner le métier du Prof. Philipp, cela ne peut sûrement pas l'être, pas plus que les descriptions étonnantes à propos de votre personnalité. Je n'ai rien dit qui aurait pu l'aider pour cela, j'ai d'ailleurs à peine parlé - elle ne m'a pas laissé placer un mot. La carte qu'elle a eue entre les mains (et qu'elle a peut-être lue adroitement sans que je m'en aperçoive) était si anodine, qu'on ne peut manquer d'être frappé par ses nombreuses remarques pertinentes à propos de votre métier et de votre tournure d'esprit. » (Freud \& Ferenczi, 1994, p. 84).

Nous remarquons ainsi que, dès l'origine, Ferenczi utilise un matériel qui concerne directement Freud lors de ses premières expériences. Dans des circonstances qui laissent à désirer sur le plan expérimental, Ferenczi exprime son étonnement après cette séance et propose quelques réflexions visant à expliquer ce qu'il a pu observer. Il imagine ainsi une « sorte d'hyperesthésie extatique à de tout petits mouvements d'expression » (Ibid., p. 85) qui permettrait d'expliquer les résultats obtenus. Cependant, il envisage également l'hypothèse de la télépathie : «Si donc, j'admets qu'elle possède vraiment des capacités inhabituelles, peutêtre pourrait-on les expliquer par une sorte de lecture de la pensée, par la lecture de mes pensées ? » (Ibid., p. 85) Ferenczi analyse plus précisément la description de la voyante :

«Mme S. ne semble jamais savoir si elle doit interpréter une idée - une image - qui émerge devant elle (elle est visuelle) dans un sens concret ou abstrait [...] Cette incertitude quant à la signification concrète ou symbolique, nous l'avons aussi dans l'interprétation des rêves. » (Ibid., p. 85)

Il propose ainsi un parallèle, récurrent dans les textes des premiers psychanalystes et des métapsychistes, concernant la proximité entre les processus mis en évidence dans les séances avec des voyants et ceux que l'on peut observer dans le rêve. Ferenczi conclut cette longue lettre sur ces quelques mots prophétiques : «Peut-être cette affaire est-elle quand même le commencement de quelque chose - et ce matériel serait alors utilisable » (Ibid., p. 86). Freud 
répond dès le lendemain, le 6 octobre 1909, par une première remarque, prudente et mesurée, à l'image de son scepticisme de l'époque : «On peut admettre en toute tranquillité que cette personne lit, avec ses yeux, ce qui lui est présenté - tout comme vous lisez cette lettre - au moyen d'un quelconque tour de passe-passe » (Ibid., p. 87). Il note cependant :

« Mais cela mis à part, il semble bien qu'il y ait quelque chose là-dedans. Le reste a l'air authentique ! [...] Mais la transmission de vos pensées par des voies incompréhensibles, voilà qui est remarquable et peut-être nouveau. Gardez le silence là-dessus, pour le moment; il nous faudra mettre en place de nouvelles expériences. » (Ibid., p. 87)

Cette lettre de Freud met en évidence dès l'origine les principaux ingrédients de leurs échanges ultérieurs : son intérêt pour la télépathie et le choix nécessaire, selon lui, d'une grande prudence sur le plan de la communication. En outre, s'il fait preuve d'un certain scepticisme, qui se traduit par l'élaboration d'explications prosaïques, il se trouve, dès ses premiers échanges avec Ferenczi, ouvert à l'existence de la télépathie. Le revirement que l'on pourra observer, en 1921, est donc en réalité le fruit d'une élaboration entamée plus de dix ans auparavant. Freud, manifestement interloqué par cette première expérience, rédige, le 11 octobre 1909, une nouvelle lettre concernant les voyances de Mme Seidler :

«Elle devine les pensées, et peut-être les pensées inconscientes de la personne qui la soumet à l'expérience - avec les malentendus et les approximations d'une sorte de déformation au passage d'une psyché à l'autre. » (Ibid., p. 88)

Freud note ainsi très tôt qu'il n'est pas tant question du contenu conscient, que du contenu inconscient, qui sera perçu et transformé par le voyant. De fait, la psychanalyse se trouve donc convoquée dans l'étude de la télépathie puisque, sans analyse du contenu inconscient échangé par transmission de pensée, on ne peut en dégager le sens véritable et donc l'origine télépathique. Cette dynamique se trouve d'autant plus complexe lorsqu'il s'agit de prendre en compte l'esprit de «l'émetteur » qui, lui aussi, peut jouer un rôle. Freud note ainsi que «dans l'analyse des oracles, il faudrait donc prendre en considération : 1/ la déformation due à la psyché ; 2/ vos propres résistances. » (Ibid., p. 88)

Freud dégage ici deux processus parallèles : les processus de déformation provenant du sujet «récepteur » mais aussi les déformations provenant de «l'émetteur». Le "récepteur» refléterait, dans une image déformée par son propre psychisme, la formation de désir inconsciente inscrite avec ses propres signifiants dans l'esprit de «l'émetteur ». Freud rejoint d'ailleurs les observations indépendantes que fera le médecin français Eugène Osty avec des voyants (Rabeyron, 2010). Pour autant, une certaine prudence est à nouveau prônée par Freud : «Pour l'instant, taisons-nous, silence absolu sur l'affaire. Le seul que j'aie mis dans la confidence est Heller ${ }^{\mathrm{iii}}$ qui, il est vrai, a fait des expériences avec elle. » (Freud \& Ferenczi, 1994, p. 88)

Ferenczi répond trois jours plus tard, le 14 octobre, en décrivant les nouvelles expériences qu'il envisage de réaliser avec l'aide de son frère comme intermédiaire, afin d'éviter une interaction directe avec la voyante. Le 8 novembre 1909, il détaille ce protocole plus raffiné, qui utilise trois lettres comme cibles. Ferenczi a occulté et modifié plusieurs détails de la première missive. La deuxième est en hongrois et ne peut donc être lue par la voyante allemande. Il l'a d'ailleurs traduite et retranscrite intégralement dans son courrier destiné à Freud. La troisième est une "fausse » lettre, rédigée par une amie et sans réelle signification affective, et placée dans une enveloppe pour plus de sécurité. Ferenczi trouve les résultats intéressants et remarque :

«Il est aussi très vraisemblable qu'elle mélange les pensées d'autres personnes avec ses propres pensées (de la façon dont nous nous le sommes expliqué après la première expérience) ; et qu'elle répète dans l'ignorance de la signification abstraite ou concrète des images qu'elle voit (et sans savoir, probablement, ce qui lui appartient et ce qui appartient au partenaire). » (Ibid., p. 104) 
Nous voyons ainsi comment la transmission de pensée amène de fait à un questionnement sur sa réalité objective, questionnement conduisant tout d'abord Freud et Ferenczi à proposer différentes hypothèses alternatives qui sont ensuite testées dans un cadre comparable aux travaux des chercheurs des «sciences psychiques » de l'époque (Méheust, 1999 ; Plas, 2000).

\subsection{Expériences avec la somnambule Mme Jelinek et empoisonnement par les huitres}

Ferenczi envisage alors une nouvelle expérience et fait allusion à une personne qui réaliserait des séances de voyance après avoir été hypnotisée. Freud, dans sa réponse du 10 novembre 1909, tempère cependant l'enthousiasme de Ferenczi. Ce dernier fait mention de nouvelles expériences, le 20 novembre, avec une médium qui se dit "somnambule», Mme Jelinek. Ferenczi lui pose plusieurs questions personnelles après qu'elle ait été placée en état de "sommeil magnétique » par son mari. Elle lui répond par quelques remarques générales. Celles-ci trouvent néanmoins un certain écho avec les préoccupations de Ferenczi. Il lui demande plus précisément: "Comment dois-je travailler, et à quoi ? », " Que convient-il de faire en ce qui concerne ma relation à Jung ? », "Que pouvez-vous me dire de mes amis viennois? ».

Par le biais de cette nouvelle expérience, et du compte rendu la concernant, Ferenczi en dit autant sur la télépathie que sur ses préoccupations de l'époque concernant Jung et Freud. On serait également tenté de faire un parallèle entre les angoisses de Ferenczi, son besoin de trouver ainsi conseil auprès d'une voyante et son désir de croire en la télépathie. Ferenczi précise également qu'il souhaiterait que Freud vienne voir Mme Jelinek, mais celuici ne répondra pas directement au contenu de cette lettre. A-t-il perçu les inquiétudes de Ferenczi à peine dissimulées derrière ce compte rendu ? Peut-être préfère-t-il, par son silence, ne pas avoir à lui répondre sur des thèmes qu'il ne souhaite pas aborder directement avec lui (en particulier la rivalité de Ferenczi avec Jung). C'est peut-être pour cette raison qu'il faudra attendre près d'un an avant de voir resurgir cette question dans leurs échanges.

C'est donc un an plus tard, le 15 novembre 1910, que ce thème réapparait dans leur correspondance. Freud décrit le cas de l'empoisonnement par les huîtres ${ }^{\text {iv }}$. Dans cette même lettre, il écrit à Ferenczi, à propos de la télépathie : "c'est bien cela que sera votre grande découverte ». L'italique vient-il insister sur le fait que Freud ne souhaite par se risquer sur ce terrain, malgré son intérêt, et qu'il laisse le soin à Ferenczi, et à Jung, d'aborder de façon approfondie le domaine de l'occulte et de la télépathie ? S'agit-il pour Freud d'un éclair de lucidité lui permettant de protéger la psychanalyse d'attaques provenant de potentiels détracteurs? Ou bien est-il davantage question d'une forme de prudence le conduisant à déléguer ainsi les tâches plus dangereuses d'une recherche qui lui paraît pourtant féconde ? Il faut savoir que Freud a d'autres conseillers, en particulier Jones et Hitschmann, beaucoup plus réticents à l'idée de suivre une psychanalyse qui se fourvoierait avec l'occultisme (Moreau, 1976 ; Jones, 1957).

Quoi qu'il en soit, on ne peut qu'être frappé de l'insistance avec laquelle Freud semble placer Ferenczi dans ce rôle de précurseur de l'étude de la télépathie, comme il a pu lui-même l'être pour l'inconscient. Mais, nous le verrons ultérieurement, il s'agit en quelque sorte d'un cadeau empoisonné, à l'image des huîtres : dans le cas où ses disciples s'éloigneraient trop de la psychanalyse, il lui serait alors possible de s'en séparer.

\subsection{Ferenczi lecteur de pensée}


Ferenczi répond dès le lendemain, le 16 novembre 1910, en décrivant un cas de transmission de pensée avec un patient homosexuel. Celui-ci semble décrire dans ses associations des échanges que Ferenczi a lui-même eu plus tôt dans la journée avec une autre personne. Ce patient dit notamment : «Le professeur Freud est vraiment un égoïste s'il compte devenir célèbre après sa mort $»$. On serait tenté une nouvelle fois de se demander dans quelle mesure Ferenczi adresse à Freud, comme avec Mme Jelinek, des propos qu'il ne peut habituellement lui tenir. Il précise :

«La façon dont mon homosexuel devine mes pensées plaide pour le fait que même des idées entièrement abstraites (c'est-à-dire déjà exprimées en images verbales) peuvent être transférées, et pas seulement des représentations imagées. » (Freud \& Ferenczi, 1994, p. 244)

Cette vision de la situation transférentielle bouleverse radicalement le cadre de l'analyse, brisant la distance entre analyste et analysant. On sait que, pour Ferenczi, l'analyse du contre-transfert est d'une importance primordiale, mais y admettre des concordances télépathiques semble un pas de franchi. Faut-il y voir la trame des essais d'analyse mutuelle de Ferenczi, où «l'hypocrisie » n'est plus possible? Ou encore, la base d'un « délire » qui l'amènera, vers la fin de sa vie, à s'imaginer faire une psychanalyse par télépathie transatlantique ? ? $^{\mathrm{V}}$ Sans enfermer la potentialité télépathique dans une "folie à deux », il est possible d'envisager sa place dans la discussion sur le cadre psychanalytique, la suggestion et la complaisance doctrinale (Ehrenwald, 1957).

Dans la même lettre, Ferenczi demande également à Freud les coordonnées d'une voyante de Munich qu'il aimerait rencontrer. Quelques jours plus tard, le 22 novembre, Ferenczi tient des propos étonnants : "Je suis un formidable voyant, ou plutôt lecteur de pensées! Je lis (dans mes associations libres) les pensées de mes patients » (Freud \& Ferenczi, 1994, p. 245). Il a en effet réalisé quatre essais avec son patient homosexuel, obtenant des «succès éclatants ». Le patient devait choisir des mots au hasard tandis que Ferenczi décrivait ses propres associations, inversant ainsi la relation habituelle analysteanalysant. Ferenczi conclut: "Cette méthode conviendra pour surprendre au travail les complexes les plus actifs des patients [...]. Je me présenterai à vous comme "astrologue de cour des psychanalystes" » (Ibid., p. 245).

Freud ne répond pas tout de suite à cette lettre, comme si, à chaque écart ou enthousiasme un peu trop marqué de son élève, il s'agissait pour lui de prendre ses distances. Trois semaines plus tard, le 2 décembre 1910, c'est donc Ferenczi qui fait une nouvelle allusion à la transmission de pensée. Il fait référence au fait que la mauvaise humeur de Freud dans ses dernières lettres semble l'avoir "contaminé », comme si elle avait "imprégné le papier ». On se demande s'il s'agit là uniquement d'un aspect métaphorique, puisque Ferenczi fait référence, dans cette même lettre, à des travaux sur la contamination par la radioactivité. Ferenczi propose également quelques nouvelles réflexions sur la transmission de pensée :

«Si on est en proie à une quelconque émotion et qu'on n'est pas tout à fait dans le coup (de l'expérience) - on peut être un bon sujet émetteur de rayonnement, mais on ne vaut rien pour la réception. Une disposition d'esprit calme est plus favorable. » (Ibid., p. 247)

Il note aussi que « les amoureux seraient plus sensibles à ces phénomènes » et ajoute :

«Le récepteur réagit à la transmission avec ses propres complexes inconscients, parmi lesquels il choisit précisément ceux qui sont les plus proches des complexes inconscients de celui qui donne les ordres. » (Ibid., p. 247)

Il illustre cet aspect théorique par un exemple tiré de la clinique obtenue avec le patient homosexuel déjà mentionné. On ne peut qu'être étonné par ce rapport entre Ferenczi et son patient homosexuel, qui semble refléter en miroir le rapport Freud-Ferenczi. Ferenczi, qui avait été, comme Jung, abusé par un homme dans son enfance, analysait-il ce problème non 
résolu avec Freud? Inversait-t-il les rôles avec ce patient homosexuel lors d'expériences de télépathie, comme il aimerait inverser les rôles dans sa relation à Freud ?

\subsection{Un destin inéluctable : qui sera l'homme de l'avenir ?}

Freud répond finalement le 3 décembre 1910 à la lettre de Ferenczi du 22 novembre. Freud partage cette fois l'enthousiasme de Ferenczi. Après ces quelques jours de réflexion, il insiste en effet sur le destin qu'il imagine pour ce dernier :

« Je vois venir le destin qui s'approche inéluctablement et je constate que c'est à vous qu'il a imparti la charge d'éclairer la mystique et autres choses de ce genre, et qu'il serait aussi vain que mesquin de vous en empêcher. » (Ibid., p. 249)

Néanmoins, cette entreprise est une nouvelle fois marquée du sceau du secret :

« Je voudrais vous inviter à accumuler les données pendant deux bonnes années encore, et en secret, et n'apparaître en public qu'en 1913, et là dans le Jahrbuch, à visage découvert. » (Ibid., p. 249).

Freud, dans un même mouvement, encourage donc Ferenczi, tout en prônant le plus grand secret. Ce dernier, qui a déjà proposé plusieurs hypothèses originales dans ses lettres précédentes, poursuit ses réflexions et précise le 19 décembre 1910 qu' «il nous faudrait quelques personnes ayant cette sensibilité ». À défaut de les trouver, il donne un exemple dans lequel il est lui-même sujet. Comme dans les cas précédents, il analyse le contenu de la transmission de pensée par le biais des déformations inconscientes et des processus décrits par la psychanalyse. Il propose également la métaphore suivante :

«Le jeu rappelle le jeu de cache-tampon des enfants; pendant les associations, le questionnant contrôle la bonne qualité ou l'inadéquation des idées qui surviennent, et il semble constamment indiquer la direction au récepteur. » (Ibid., p. 254)

Freud annonce alors à Ferenczi, le 29 décembre 1910, qu'il a mis Jung dans la confidence à propos de la transmission de pensée. Ce dernier s'est d'ailleurs dit déjà convaincu. Freud propose donc à Ferenczi d'« introduire Jung dans ses calculs » et précise au passage, à propos de celui-ci : «Je suis plus que jamais convaincu qu'il est l'homme de l'avenir ». Si Ferenczi signifie sagement son obéissance, dans le post-scriptum d'une lettre rédigé le 3 janvier ( « je vais bientôt entreprendre Jung avec la transmission de pensée »), cette question de l'occulte, et de la transmission de pensée, semble condenser au plus haut point les rivalités entre Ferenczi et Jung, rivalités qui se voient attisées par les remarques précédentes de Freud. Par la suite, Freud confia à Jung (lettre du 15 juin 1911) qu'il était très impressionné : les expériences de Ferenczi l'ont « rendu humble et brisé ses présomptions à l'encontre de l'occultisme ». Un peu plus tard (le 10 janvier 1912), toujours dans sa correspondance avec Jung, il considère que les preuves de Ferenczi à l'appui de la transmission de pensée sont une des deux contributions à la psychanalyse les plus importantes des derniers temps. Ce faisant, Freud comblait certaines des attentes de Jung, tout en faisant de Ferenczi le fils prodigue. Qui sera le véritable héritier du maître, celui qui saura réellement dégager quelques lumières sur l'occultisme, avec tous les enjeux narcissiques que cela représente?

Par la suite (3 janvier 1911), Freud décrira la prophétie des jumeaux ${ }^{\mathrm{vi}}$. Il rapporte en détail le dialogue avec sa patiente et, le 24 janvier 1911, propose un nouvel exemple, succinct, de transmission de pensée et de déformation du message transmis.

\subsection{L'étrange inquiétant et le soldat Kohn}


C'est ensuite le 3 mai 1911 que Ferenczi expose une expérience étonnante dont il est le sujet. Alors qu'il se trouve dans le bus, il tente de deviner les pensées de plusieurs passagers, en particulier celles d'un soldat. Après quelques associations, il acquiert la certitude que ce soldat se nomme «Kohn ». Pour vérifier son intuition, Ferenczi va jusqu'à lui demander à la descente du bus si son nom est bien Kohn. Le soldat, surpris, lui répond qu'il s'appelle bien «Johann Kohn » et lui demande comment ils se connaissent. Ferenczi précise que cette trouvaille l'a laissé «tout rêveur»! Dans sa réponse, Freud trouve cette histoire " étrangement belle » (unheimlich schön), mais se demande néanmoins s'il ne pourrait pas s'agir d'une cryptomnésie, car il ne croit pas qu'une transmission de pensée puisse être aussi précise. Il fait également une nouvelle fois allusion au territoire de l'occulte, à Jung, et au fait que «ce sont des expéditions dangereuses, et là, je ne peux pas vous accompagner » (Freud \& Ferenczi, 1994, p. 289). Freud termine sa lettre ainsi : «Je vous salue, vous l'étrange inquiétant. »

Le 13 mai, Ferenczi explique en détail les raisons pour lesquelles la cryptomnésie ne lui paraît guère probable. Freud se dit impressionné par les remarques du psychanalyste hongrois, écartant la cryptomnésie, et ajoute que cette histoire du « petit Kohn » est « un point de départ qui peut mener à quelque chose » (Ibid., p. 293). Le 11 octobre 1911, Ferenczi fait une brève allusion à la Society for Psychical Research ${ }^{\text {vii }}$ qui demande à Freud de lui renvoyer une déclaration d'adhésion, ce qu'il fera effectivement. Puis, il faut attendre janvier 1912 pour que Freud indique avoir rédigé un texte pour la revue de la SPR intitulé «A note on the Unconscious in Psycho-Analysis » (Freud, 1912).

\subsection{Séparation d'avec Jung et expériences avec Alexander Roth}

La fréquence des échanges de Ferenczi et Freud sur ce sujet va alors diminuer durant quelques mois pour deux raisons. La première est que Ferenczi semble en difficulté face à ses interminables hésitations matrimoniales. Celles-ci occupent donc la plupart de ses pensées et de ses lettres. La deuxième raison est la tournure que prennent les événements avec Jung. Alors que celui-ci devait travailler de concert avec Ferenczi dans l'exploration scientifique de l'occulte, il dévie de sa route. Freud et Ferenczi échangent ainsi fréquemment concernant leur déception face à l'évolution théorique de Jung. Ferenczi commente dans une lettre du 12 mai 1913 : « enfin, je suis parvenu au sens le plus secret du travail de Jung. C'est tout simplement sa profession de foi occultiste, déguisée sous des dehors scientifiques. » (Freud \& Ferenczi, 1994, p. 513)

Ce n'est que le 23 novembre 1913 que Freud rédige une nouvelle lettre abordant la question de la transmission de pensée, suite à une conférence de Ferenczi sur ce sujet à la Société psychanalytique de Vienne quatre jours avant. Il indique avoir convié le professeur Alexander Roth, avec son épouse, à l'Association viennoise de psychanalyse pour des expériences de transmission de pensées (Ibid., p. 248). Il fait référence à une séance effectuée l'après-midi même en présence de Rank, Sachs, Hitschmann et leurs familles respectives. Freud juge cette session " lamentable » car elle n'a même pas mené à « l'ombre d'un succès » et se montre extrêmement critique. Dans une lettre du 27 novembre, il demande à Ferenczi de récupérer l'attestation que ce dernier avait transmise à Alexander Roth après quelques expériences :

« Songez seulement que si les choses en viennent à une discussion publique concernant cet homme, qui dès maintenant fait état à Vienne de ses succès à l'Association Psychanalytique, nous devrions vous lâcher. » (Ibid., p. 557)

Freud craint-il que Ferenczi s'égare de la même façon que Jung ? Ferenczi, dans une lettre du 29 novembre, renonce alors à la publication d'un article sur la transmission de 
pensée. Il transmet cependant à Freud un communiqué qui résume sa récente conférence sur ce thème. Ceci constitue leur dernier échange à ce propos avant la Première Guerre mondiale et clôt leur période de correspondance la plus prolifique sur ce sujet.

Entre 1914 et 1919, leurs échanges sur la transmission de pensée sont en effet moins nombreux. Ferenczi, dans une lettre datée du 22 novembre 1914, fait une brève remarque concernant une prédiction de Jung. Elle fait écho au décès du frère de Freud, Emmanuel. Freud reproche à Ferenczi de penser à cette prédiction et lui dit: "Vous êtes bien plus profondément plongé dans l'occulte que nous ne le pensions » (Freud \& Ferenczi, 1996a, p. 40). Ferenczi répond, le 30 novembre 1914, que son occultisme « est très nettement séparé du reste du savoir et ne le perturbe en aucune manière » et qu'il est ainsi «libre de tout mysticisme » (Ibid., p. 40).

C'est ensuite Freud qui aborde à nouveau ce sujet durant l'été 1915, le 10 juillet plus exactement, évoquant un "rêve prophétique » dans lequel ses fils étaient tués, à commencer par Martin. Freud précise avoir appris, le lendemain, qu'un garçon qui avait sauvé son fils venait de trouver la mort lui-même (Ibid., p. 76). Le 21 juillet, Freud apprend également, par une lettre, que son fils va bien, la prophétie ayant donc « échoué ». Cependant, ce dernier a vu la balle d'une patrouille russe érafler son bras droit et, si Freud dit ne pas connaître la date exacte de l'événement, il reconnaît « qu'on est probablement plus sensible la nuit » (Ibid., p. 81).

Ferenczi répond dix jours plus tard : «Je suis convaincu de l'existence de l'induction de pensées. Ainsi, je crois même que la preuve que les prophéties sont possibles ne pourrait ni ne devrait forcer personne à abandonner la base scientifique ». Il précise également que ce n'est pas l'occulte qui l'intéresse mais plutôt une "désocculation", probablement fondée, en dernière instance, "sur certaines tendances magico-religieuses " dont il "se défend en voulant apporter la lumière sur ces choses. » (Ibid., p. 82). Le 16 décembre 1915, Ferenczi explique cette fois qu'une tireuse de cartes lui a "prophétisé » qu'il se marierait deux fois. Elle lui a également annoncé qu'il aurait dû se marier "récemment» mais "qu'il avait repoussé sa fiancée » (Ibid., p. 109).

Les échanges entre Freud et Ferenczi se raréfient ensuite, mais leurs quelques courriers penchent en faveur de la réalité de la télépathie, au point que cette question ne semble plus faire débat entre eux. C'est le 18 août 1921 que Freud annoncera avoir terminé de rédiger «Psychanalyse et télépathie ». Le 19 février, Freud, dans une lettre circulaire, fait référence aux expériences récentes du Professeur Gilbert Murray publiées dans les Proceedings de la SPR de décembre 1924 et écrit qu'il est « grand temps de discuter de ce problème ». Ferenczi fait pour sa part allusion à Myers (1903) et à sa tentative d'expliquer la télépathie à l'aide du subliminal. Il observe ainsi que :

«Mes propres cas sont significatifs non seulement comme confirmation de l'effectivité de la transmission de pensée, mais comme une sorte de preuve objective des modes opératoires de l'Ics, proposés par la psychanalyse, notamment de la symbolique. » (Freud \& Ferenczi, 1996b, p. 210)

Ferenczi demande à Freud l'article de la SPR. Il explique que de nombreux écrits sur la télépathie ont été produits récemment par les «outsiders» des milieux de sciences psychiques. Dans cette lettre, Ferenczi réagit à la circulaire de Freud datant de mi-février dans laquelle ce dernier explique que :

«La plus forte impression littéraire de ce mois m'est venue d'un rapport sur des expériences de télépathie avec le professeur Murray. Je reconnais que l'impression de ce rapport a été tellement forte que je suis prêt à abandonner mon refus de l'existence de la transmission de pensée [...] Je serais même prêt à offrir à la cause de la télépathie le soutien de la psychanalyse. » (Ibid., p. 210)

Freud aimerait publier « l'article secret » comprenant différents cas de télépathie, mais il ne le peut pas, «pour cause de discrétion médicale», car il faudrait que les «deux 
destinataires des prophéties non accomplies » décèdent avant lui (Ibid., p. 229). C'est ensuite le 15 mars 1925 que Ferenczi fait à nouveau allusion à quelques expériences de télépathie «passablement réussies » avec Freud et sa fille Anna, qui possédait, selon son père, la « sensibilité télépathique ». Une circulaire de Freud y fait d'ailleurs aussi référence :

«Ferenczi était chez nous pour un dimanche. Nous avons discuté de bien des choses et fait, à trois, des expériences de transmission de pensée qui ont remarquablement bien réussi, en particulier l'expérience où j'ai moi-même joué le rôle de médium en complétant ensuite mes idées sur un mode analytique. Nous arrivons à serrer l'affaire de plus près. » (Ibid., p. 230)

On peut lire une dernière allusion à ce sujet dans une circulaire du 18 avril 1925, dans laquelle Ferenczi précise :

«Pour la question de la transmission de pensée, je soutiens mon point de vue, mais, en accord avec Monsieur le Professeur, je renonce pour l'instant à publier mes acquis, expériences et tentatives d'explication. » (Ibid., p. 238)

La question a donc, à ce moment, quitté la dyade Freud-Ferenczi pour concerner plus largement un petit cercle d'analystes.

\section{Destin et modernité de l'occulte dans la correspondance Freud-Ferenczi}

\subsection{Reprise dans la questions de l'occulte dans le champ psychanalytique}

Dès lors que ces questionnements ont été partagés au-delà des échanges de Freud et Ferenczi, ils se sont progressivement disséminés dans le champ psychanalytique. Georges Devereux a ainsi réuni 31 textes portant sur ce sujet dans un ouvrage intitulé Psychoanalysis and the Occult publié en 1953. Dans cette anthologie, nous retrouvons les textes de Freud traduits en anglais mais également ceux d'autres analystes avec, entre autres, Deutsch, Burlingham, Fodor ou Hollós ${ }^{\text {viii }}$. On trouve également des débats épistémologiques de haut vol, en particulier avec Ellis (qui deviendra par la suite l'un des initiateurs des thérapies cognitives). L'occulte, et plus précisément le transfert de pensée (Evrard et Rabeyron, sous press), se situe ainsi à la croisée d'épistémologies différentes. Objet à la fois de fascination et de rejet, il demeurera en marge des réflexions des théories psychanalytiques et tombera ainsi en désuétude jusqu'au début des années 1980, période lors de laquelle les textes de Freud sur ce sujet ont été traduits en français, suscitant un renouveau d'intérêt pour ce thème parmi les psychanalystes (Moreau, 1976 ; Rey et Granoff, 1983).

L'étude détaillée de cette correspondance offre encore aujourd'hui une perspective intéressante sur des thèmes qui nous paraissent toujours d'actualité. Ils représentent en quelque sorte les prémisses historiques permettant de relancer certains questionnements fondamentaux de l'approche clinique, qu'il s'agisse de l'intersubjectivité précoce (FisherBernardino, 2005), des processus de co-pensée (Widlöcher, 1996) ou des relations transférocontre-transférentielles, autant de thèmes chauffés à blanc par la clinique de l'occulte. Cette correspondance, et les textes de Freud sur ce thème, apparaissent également comme les premières tentatives de théoriser d'un point de vue psychanalytiques certaines expériences jugées «occultes » à l'époque, tentatives qui peuvent s'avérer pertinentes pour penser le champ clinique contemporain des expériences vécues comme «paranormales », ou exceptionnelles $^{\text {ix }}$. Ces dernières années ont en effet vu émerger un certain nombre de publications sur ce thème aussi bien en France qu'à l'étranger (Eschel, 2006 ; Laufer, 2007 ; Evrard, 2010), dans une perspective psychanalytique qui n'est pas limitée aux concordances d'apparence télépathiques dans l'analyse d'adultes, mais explore également plus largement la possession (Juranville, 2001), les expériences de sortie du corps (Le Maléfan, 2011), les 
expériences de mort imminente (Le Maléfan, 2010) ou encore l'impression d'avoir été « enlevé » par des extraterrestres (Maleval \& Charraud, 1997).

\subsection{Conséquences transféro-contre-transférentielles de l'occculte}

Au-delà des recherches menées dans la continuité des réflexions initiées dans cette correspondance, le traitement de l'occulte par Freud et Ferenczi permet d'observer l'impact que le traitement de l'occulte a pu avoir sur eux et sur leur relation, ce qui fait de ces échanges une forme de «révélateur» des processus contre-transférentiels engendrés par ces expériences de façon plus générale. Ainsi, dans l'ensemble de la correspondance, on remarquera tout d'abord de quelle manière l'occulte peut s'apparenter à des processus de nature défensive. Ferenczi exprime en effet à travers plusieurs exemples ses craintes concernant la rivalité avec Jung et son avenir matrimonial. Nous pouvons de la même façon interpréter certaines «transmissions » comme étant l'expression d'une forme d'agressivité inconsciente à l'égard de Freud. Les propos de Ferenczi semblent cependant « masqués » sous couvert de transmission de pensée, comme s'ils étaient exprimés entre parenthèses par une formulation du type : «ces pensées ne viennent pas de moi, elles proviennent simplement d'une perception télépathique de la réalité ». Dans cette perspective, l'occulte apparaît comme une tentative de mettre à distance des dynamiques inconscientes tout en les exprimant, rejoignant ainsi des hypothèses proposées par Gori (1996). Il est ainsi fréquent que l'occulte, et plus précisément les «transferts » de pensée, soient l'expression déguisée et détournée de motions pulsionnelles inconscientes. Ce que Freud a pu nommer comme le «corps étranger» de la télépathie représenterait alors des matériaux refoulés et transférés dans ce contournement dynamique des conflits.

Nous pouvons également relever dans la même perspective les liens subtils entre occultisme et enjeux narcissiques. Freud fait notamment allusion à une « grande découverte », laissant entrevoir, tout comme Ferenczi, une fascination certaine pour ce sujet. On retrouve fréquemment, de la même façon, dans la clinique des expériences exceptionnelles, cet investissement narcissique qui se traduit fréquemment par l'impression qu'ont les personnes qui rapportent de telles expériences d'avoir vécu quelque chose «d'extraordinaire » et d'être donc « différentes ». Il y a ainsi la rencontre entre un vécu incompréhensible voire impensable de prime abord, et la structuration psychique d'un individu, ce à quoi font écho les «tendances magico-religieuses » décrites par Ferenczi.

Associés à cet aspect narcissique, on trouve fréquemment des vécus de l'ordre non seulement de l'inquiétante étrangeté, mais également du registre du secret. Ces deux particularités sont très présentes dans ces lettres. Freud trouve Ferenczi parfois «inquiétant » et, si le sujet semble les passionner tous deux, il est également marqué du sceau du secret car Freud interdit à Ferenczi de publier sur ce thème tout en lui proposant de continuer ses recherches. Cette dissimulation, souvent liée à la honte, est également fort présente dans la clinique des expériences exceptionnelles. La peur de la folie n'est généralement pas bien loin. De plus, le «paranormal » - de par son positionnement sociologique hors de la «normalité »- semble ainsi donner aux personnes qui vivent de telles expériences le sentiment coupable de la rencontre avec l'interdit.

Ces trois caractéristiques (fascination, inquiétante étrangeté, secret) qui composent fréquemment la mosaïque contre-transférentielle des expériences exceptionnelles transforment parfois l'occulte en un objet qui pourrait en apparence « contaminer » ceux qui le traitent. Cette idée de contamination prend sa source dans le lien étroit entre l'occulte et les couches les plus archaïques du psychisme, dans des zones où les distinctions dedans/dehors, réalité interne/réalité externe, deviennent plus floues, et où la raison tend à perdre pied face à 
des enjeux inconscients plus obscures. Nous retrouvons cet aspect à travers la dynamique qui s'instaure autour de l'évolution de Jung, dont Freud et Ferenczi disent craindre l'égarement dans le mysticisme. Une fois Jung écarté, c'est au tour de Ferenczi d'avoir à prouver de façon récurrente à Freud qu'il est bien dans une tentative rationnelle de «dés-occulation de l'occulte». Il est important de prendre en compte cette particularité pour cette clinique spécifique afin de mieux pouvoir s'en détacher, et parvenir à penser ces expériences comme n'importe quelle expérience humaine. Ainsi, comme le remarque Devereux (1953), c'est notamment de la capacité qu'aura le clinicien à garder une position de neutralité, sous la forme d'une position d'épochè, qu'il lui sera possible de ne pas sombrer dans la crédulité, héritière de la pensée magique, ou à l'inverse, dans le rejet a priori, souvent assimilable à une formation réactionnelle face à cette même pensée magique. Freud avait également analysé dans le détail ce type de positionnement dans «Rêve et occultisme », en proposant toute une série de réflexions épistémologiques introduisant son article le plus abouti sur l'occultisme (Freud, 1932).

\subsection{Processus inconscients et intersubjectivités dans l'occulte}

Au-delà de ces aspects transféro-contre-transférentiels, une autre dimension de cette correspondance retient en particulier notre attention, à savoir l'étude que font Freud et Ferenczi de la nature des messages «transférés ». On remarquera tout d'abord que l'analyse des expériences effectuées avec des voyants (ou sur eux-mêmes) les conduit à mettre en évidence des processus identiques à ceux du rêve. Le «message» semble ainsi subir un ensemble de processus de déformation qui expliquerait l'aspect abstrait et métaphorique de la transmission de pensée. Ferenczi met également en évidence plusieurs facteurs favorisants : la prise en compte des émotions, du lien amoureux, des résistances de l'émetteur et le fait que certains sujets semblent plus doués que d'autres.

Ferenczi note ainsi que la télépathie pourrait faire office de «preuve objective » des modes opératoires de l'inconscient, en particulier au niveau symbolique. Dans cette perspective, ces études peuvent être considérées comme des préludes aux travaux contemporains sur la transmission psychique inconsciente et, plus largement, sur les processus de l'intersubjectivité. En effet, Freud et Ferenczi ne semblent pas être en mesure, à l'époque, de saisir la complexité, la profondeur et la précision des processus intersubjectifs en jeu. Ils ne peuvent donc concevoir de telles interactions que sur le mode de la transmission de pensée. On retrouve fréquemment le même mode de réaction chez des personnes confrontées à des expériences qu'elles considèrent comme étant de nature télépathique ou prémonitoire. Le niveau de précision, la particularité et l'étrangeté des processus en jeu, la profondeur et la force des processus intersubjectifs qu'elles impliquent, leur semblent souvent inconciliables avec des explications prosaïques. La force des processus inconscients, et plus précisément de la transmission psychique inconsciente (Ciccone, 1999), demeure ainsi souvent profondément énigmatique pour ces personnes. Les théories actuelles de la co-pensée (Widlöcher, 1996) et de la co-psychéité (Georgieff, 2008), que nous ne détaillerons pas ici, offrent ainsi de nouvelles pistes pour saisir la nature de ces processus intersubjectifs, aussi bien dans le cadre de l'analyse que lors d'expériences spontanées du quotidien

C'est probablement cette particularité qui conduit également aux questionnements récurrents de Freud et Ferenczi concernant la réalité objective de la télépathie. Sans preuve expérimentale de cette dernière, semblent-ils se demander, comment prendre en compte son éventuelle influence en psychanalyse ? Cette interrogation les conduit à étudier et à écarter plusieurs explications, en particulier l'hyperesthésie, la fraude et la cryptomnésie. Ils effectuent également plusieurs expérimentations au cours desquelles ils sont eux-mêmes sujets et se trouvent alors confrontés aux mêmes difficultés que les métapsychistes de l'époque (Rabeyron, 2010). Ils obtiennent des succès surprenants, qui atteignent leur apogée 
avec la «clairvoyance» spontanée de Ferenczi concernant le soldat Kohn. Mais ils découvrent aussi les difficultés d'observation et de reproductibilité relatifs à ce sujet. Ce manque de fiabilité dans les résultats obtenus fait alors courir un grand risque à la psychanalyse, risque que Freud ne veut pas prendre. Ce questionnement sur l'aspect véridique de ces expériences nous parait là encore être une caractéristique fréquente de ces expériences qui rend leur compréhension d'autant plus complexe. Là encore, cette correspondance est d'actualité puisque l'on retrouve encore aujourd'hui tout un ensemble de travaux expérimentaux (Storm, Tressoldi \& Di Risio, 2010) et de recherches psychanalytiques (Mayer, 2001) qui interrogent la nature de certaines de ces expériences.

\section{Conclusion}

Il nous semble en définitive que la prise en compte des différents aspects relatifs à la clinique de l'occulte, tels qu'ils sont travaillés dans le détail tout au long de la correspondance entre Freud et Ferenczi, représente un outil essentiel pour mieux saisir et se représenter certains des processus impliqués dans les expériences exceptionnelles. En outre, loin de mettre en évidence une éventuelle naïveté chez Freud et Ferenczi, ces échanges montrent au contraire leur souci du détail, l'ouverture dont ils ont su faire preuve de même que les tentatives de compréhension et de théorisation qu'ils ont engagées, dans les limites des bornes épistémologiques de leur époque. La profondeur de ce thème, aussi original que complexe, nous paraît ainsi loin d'être épuisé. Plusieurs des hypothèses et des réflexions issues de cette correspondance et d'autres écrits psychanalytiques sur l'occulte représentent en effet une voie de recherche pouvant encore conduire aujourd'hui à des perspectives novatrices et possiblement pertinentes pour le champ de la psychanalyse et de la psychologie clinique.

\section{Bibliographie}

Bonomi C. (2000), L'allégation de Jones concernant la détérioration mentale de Ferenczi : une réévaluation, Filigrane, 53-61.

Ciccone A. (1999). La transmission psychique inconsciente: identification projective et fantasme de transmission, Paris, Dunod.

Devereux G. (1953), Psychoanalysis and the occult, New York, International University Press.

Ehrenwald J. (1957), The telepathy hypothesis and doctrinal compliance in psychothrepay, American Journal of Psychotherapy, 11, 359-379.

Evrard R. (2010), Psychiser le Maître absolu : solutions pubertaires par le paranormal. Adolescence, 28(4), 841-854.

Evrard R. et Rabeyron T. (sous press), Les psychanalystes et le transfert de pensée, L'Evolution Psychiatrique.

Eschel O. (2006), Where are you, my beloved ? On absence, loss, and the enigma of telepathic dreams, International Journal of Psychoanalysis, 87(6), 1603-27.

Ferenczi S. (1899), Spiritism, Psychoanalytic Review, 50, 139-144, 1963.

Ferenczi S. (1912), Un cas de "déjà-vu", In Euvres complètes (pp. 210-222), Paris, Payot, 1968.

Fisher Bernardino L. (2005), Un retour à Freud pour fonder la clinique psychanalytique des bébés et de leurs parents : les études sur la télépathie, Figures de la Psychanalyse, 11.

Fodor N. (1979), Les aventures psychiques de Ferenczi, Le Coq-Héron, 73, $20-32$. 
Fortune C. (2007), Sandor Ferenczi's Analysis of « R.N. »: A Critically Important Case in the History of Psychoanalysis, British Journal of Psychotherapy, 9(4), 436-443.

Freud S. (1899), Une prémonition onirique accomplice, In Résultats, idées, problèmes I (pp. 109-111), Paris, PUF, 1984.

Freud S. (1901), Psychopathologie de la vie quotidienne, Paris, Payot, 2004.

Freud S. (1912), A note on the Unconscious in Psycho-Analysis, Proceedings of the Society for Psychical Research, 26, 312-318.

Freud S. (1914), Déjà-rencontré dans l'analyse, In La technique psychanalytique (pp. 72-79), Paris, PUF, 1970.

Freud S. (1925), Quelques suppléments à l'interprétation des rêves, In Euvres complètes, XVII (pp. 185-188), Paris, PUF, 1992

Freud S. (1932), Rêve et occultisme. In Nouvelles conférences d'introduction à la psychanalyse (pp. 45-79), Paris, Gallimard, 1984.

Freud S. (1936), Un trouble de mémoire sur l'Acropole, Lettre à Romain Rolland, In Résultats, idées, problèmes II (pp. 221-230), Paris, PUF, 1985.

Freud S. \& Ferenczi S. (1994), Correspondance, 1908 - 1914, Paris, Calmann-Levy.

Freud S. \& Ferenczi S. (1996a), Correspondance, 1914 - 1919, Paris, Calmann-Levy.

Freud S. \& Ferenczi S. (1996b), Correspondance, 1920 - 1933, les années douloureuses. Paris, Calmann-Levy.

Georgieff N. (2008), L'empathie aujourd'hui : au croisement des neurosciences, de la psychopathologie et de la psychanalyse, La psychiatrie de l'enfant, 51(2), 357-393.

Gori R. (1996), Pensées de transfert ou transfert de pensée, In La preuve par la parole : Sur la causalité en psychanalyse (pp. 51-73), Paris, PUF.

Granoff W. \& Rey J. M. (1983), L'occulte, objet de la pensée freudienne, Paris, PUF.

Jones E. (1957), La vie et l'œuvre de Sigmund Freud (3 tomes), Paris, PUF, 1969.

Juranville A. (2001), Figures de la possession : actualité psychanalytique du démoniaque, Grenoble, PUG.

Lacan J. (1973-1974), Les non-dupes errent, Séminaire non publié.

Laufer L. (2007), De l'image revenante aux illusions bénies (expériences de spiritisme), Champ Psychosomatique, 46, 65-78.

Le Maléfan, P. (2010), La mort imminente et l'hallucinatoire salutaire, Clinique du deuil de soi anticipé, Etudes sur la mort, $\mathrm{n}^{\circ} 137$.

Le Maléfan P. (2011), La « sortie hors du corps » comme nouveau tropisme pour la clinique du corps ?, Recherches en Psychanalyse, 11.

Maleval J.-C. \& Charraud N. (1997), Modernité du démoniaque, Psychologie clinique, 4, 117 130

Mayer E. L. (2001), On «telepathic dreams ? » : an unpublished paper by Robert J. Stoller, Journal of the American Psychoanalytic Association, 49, 629-657.

Méheust B. (1999), Somnambulisme et médiumnité (2 tomes), Paris, Les Empêcheurs de Penser en Rond.

Moreau C. (1976), Freud et l'occultisme, Paris, Privat.

Plas R. (2000), Naissance d'une science humaine, la psychologie: les psychologues et le "merveilleux psychique », Rennes, PUR.

Onfray,M. (2010), Le crépuscule d'une idole, l'affabulation freudienne, Paris, Grasset.

Rabeyron T. (2009), Les expériences exceptionnelles : entre neurosciences et psychanalyse, Recherches en Psychanalyse, 8. 
Rabeyron T., Chouvier B. \& Le Maléfan P. (2010), Clinique des expériences exceptionnelles : du trauma à la solution paranormale, L'Evolution Psychiatrique, 75(4), 633-653.

Rabeyron T. (2010), Review of La connaissance supranormale, étude expérimentale by Eugène Osty, Journal of Scientific Exploration, 24(2), 351-357.

Storm L., Tressoldi P.E \& Di Risio L. (2010), Meta-analysis of free-response studies, 19922008 : assessing the noise reduction model in parapsychology, Psychological Bulletin, 136(4), 471-485.

Turnheim M. (2008), Freud le médium (Notes sur l'affaire de la télépathie), Psychanalyse, 12, 41-53.

Walter G. (1930), Lettre à Eugène Osty du 15 octobre 1930, Archives de l'Institut Métapsychique International, Dossier 22 (3).

Widlöcher D. (2004), The third in mind, Psychoanalytic Quarterly, 73, 197-213.

i Recherches sur le spiritisme, l'hypnotisme et les phénomènes «paranormaux » comme objets de la psychologie naissante et initiées notamment par des universitaires anglosaxons à la fin du XIXème siècle et au début du XXème siècle (Pour plus de détails, voir notamment : Plas, 2000)

ii Lacan, dans sa leçon inédite du 20 novembre 1973, lors de laquelle il fut interrogé à propos des «phénomènes psi », interpréta ce « don physiologique » comme relevant du mythe d'un «corps plus calé », manière de faire entendre comment l'occulte fut d'abord situé par Freud à l'extérieur du domaine de l'inconscient.

iii Hugo Heller (1870-1923), hongrois de naissance, était le deuxième éditeur viennois des travaux de Freud et des principales revues de psychanalyse de l'époque. Il appartenait au premier cercle des psychanalystes viennois.

Un étudiant analysé par Freud avait consulté une voyante-astrologue qui prophétisa la mort de son beau-frère par empoisonnement suite à l'ingestion d'écrevisses ou d'huîtres. Le beau-frère avait effectivement manqué de mourir d'une intoxication suite à un repas d'huîtres, mais un an auparavant. Freud interprète qu'une rivalité refoulée (à l'égard de la sœur de l'étudiant) s'est exprimée au travers de cette semi-prophétie, prise comme un transfert de pensée entre l'étudiant et la voyante (Freud, 1921).

Jones (1957, 3ème volume, p. 460) a soutenu cette allégation d'un délire télépathique de Ferenczi, s'appuyant sur la lettre que lui a envoyé Freud le 29 mai 1933, où ce dernier prétend que Ferenczi croyait que sa patiente lui envoyait des vibrations par-delà l'océan, une influence qu'il ressentait comme bénéfique voire salvatrice. Néanmoins, Jones a multiplié les allégations infondées sur la «folie de Ferenczi » (Bonomi, 2000). La patiente en question est la célèbre Mrs Elizabeth Severn, alias R.N. dans le Journal clinique de Ferenczi (1932), avec qui il avait développé une analyse mutuelle à partir de 1924 (Fortune, 2007). Or, il se peut que Freud ait inversé les données du problème car c'est Mrs Severn qui prétendait être médium. (Elle s'est ainsi présentée au Dr Osty lors d'une visite à l'Institut Métapsychique International de Paris le 20 octobre 1930 ; cf. Walter, 1930). Déjà, bien avant de connaître Ferenczi, sa personnalité problématique l'avait amené à se faire alchimiste, voyante, guérisseuse et « psychothérapeute métaphysique ». Elle prétendait dans ses ouvrages pratiquer la «thérapie télépathique », une technique que Ferenczi ne théorisera jamais.

vi Un homme écrit à Freud car il a rêvé que sa seconde femme, avec laquelle il n'a plus de relations sexuelles et avec qui il ne souhaite plus avoir d'enfants, aurait des jumeaux, au moment même où un télégramme lui a été envoyé pour lui annoncer que sa fille avait mis au monde des jumeaux. Freud (1932) interprète ce rêve comme un message télépathique reçu en rêve et déformé par un mécanisme de déplacement, si bien que, dans ce cas, indique Freud, 
« la psychanalyse a découvert un état réel de choses que nous n'aurions pas trouvé sans son concours ».

vii La Society for Psychical Research est un organisme de recherche fondé en 1882 par des universitaires de Cambridge, notamment Frederic Myers, et dont le but était l'étude scientifique des phénomènes dits «paranormaux ». Elle sera notamment présidée par Henri Bergson et sa branche américaine, l'American Society for Psychical Research, sera fondée par William James. Freud sera membre de la SPR de 1911 à 1938 et recevait les publications de cette société. Il saisira l'occasion d'y publier un article pour démarquer l'inconscient des notions de subconscient et de subliminal, afin de préciser la frontière avec les théories de Myers (1903). Ce dernier fut pourtant l'un des premiers à faire connaître les travaux de Freud en Grande-Bretagne.

vii Moreau (1976) distingue une «école hongroise » de psychanalystes intéressés par le transfert de pensées, avec comme possibles héritiers de cette question qui travaillait Ferenczi : Hollós, Róheim, Balint, Hann-Kende, Fodor et Devereux.

ix Clinique que nous rencontrons en particulier au sein d'un service de consultation spécialement dédié aux expériences exceptionnelles (Pour plus de détails, cf. Rabeyron, Chouvier \& Le Maléfan, 2010) 